\title{
A highly elastic and moldable polyester biomaterial for cardiac tissue engineering applications
}

${ }^{1,2}$ Locke Davenport Huyer, ${ }^{1,2}$ Boyang Zhang, ${ }^{1,2}$ Anastasia Korolj, ${ }^{1,2}$ Miles Montgomery, ${ }^{3}$ Stasja Drecun, ${ }^{1,2}$ Genevieve Conant, ${ }^{1}$ Yimu Zhao, ${ }^{2}$ Lewis Reis, ${ }^{1,2 *}$ Milica Radisic

${ }^{1}$ Department of Chemical Engineering and Applied Chemistry, University of Toronto, Toronto, ON, Canada

${ }^{2}$ Institute of Biomaterials and Biomedical Engineering, University of Toronto, Toronto, ON, Canada

${ }^{3}$ Human Biology, University of Toronto, Toronto, ON, Canada

Correspondence to:

*164 College St, Rm 407 Toronto, ON, M5S 3G9, m.radisic@utoronto.ca

\section{SUPPLEMENTARY INFORMATION}

8 pages, 5 figures 


\section{Scanning Electron Microscopy of Porous Structures:}
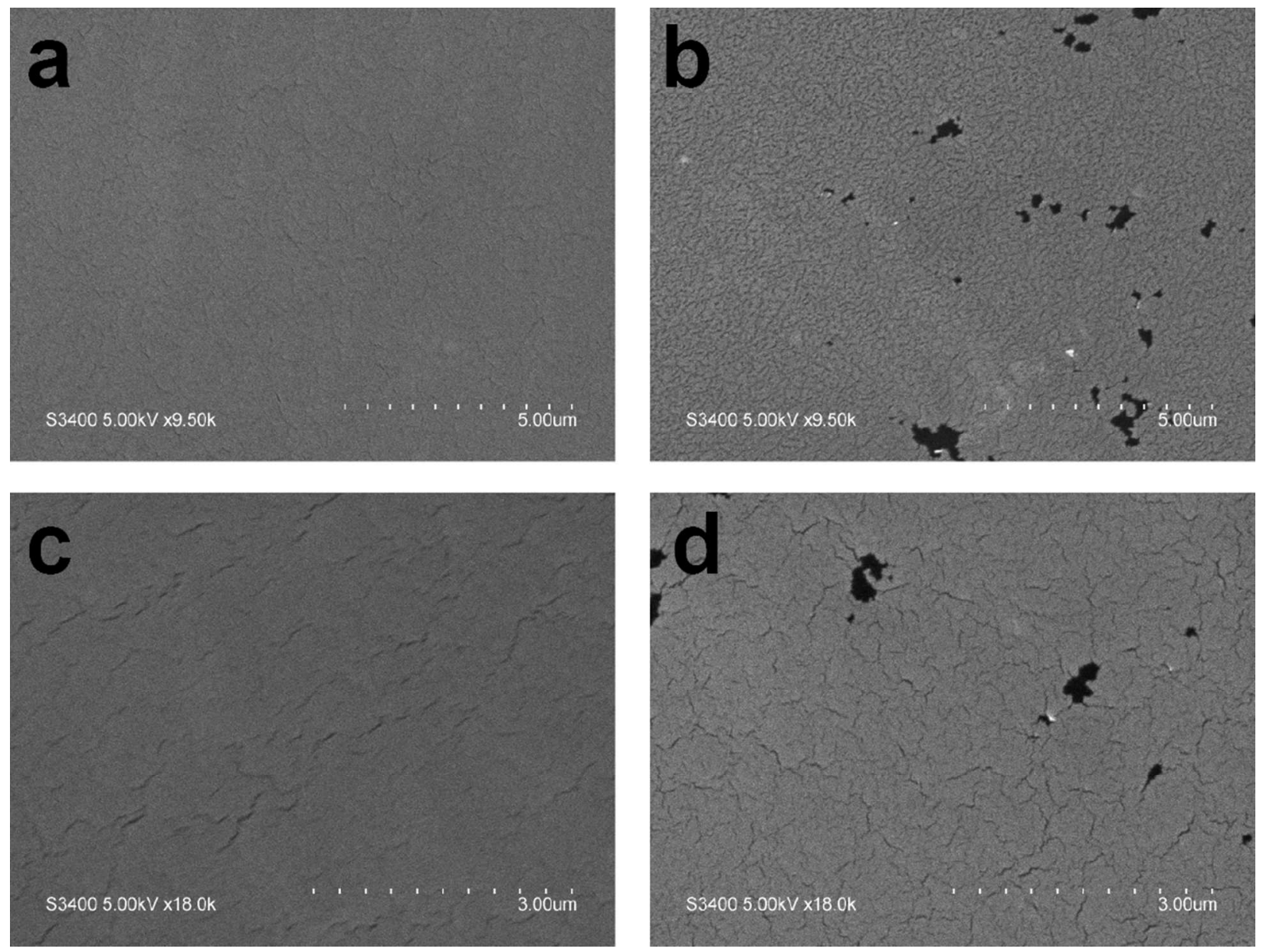

Figure S1: SEM images of non porous $(a, c)$ and nanoporous $(b, d) 124$ polymer structures. There is an observed increase in the density of micropores in the porous samples, suggesting the increased mass loss from the polymer bulk with the leaching of initial PEGDM porogen content. Pores in the samples without initial porogen content are attributed to solubilization of low molecular weight polymer chains. 


\section{Change in Young's Modulus over time:}
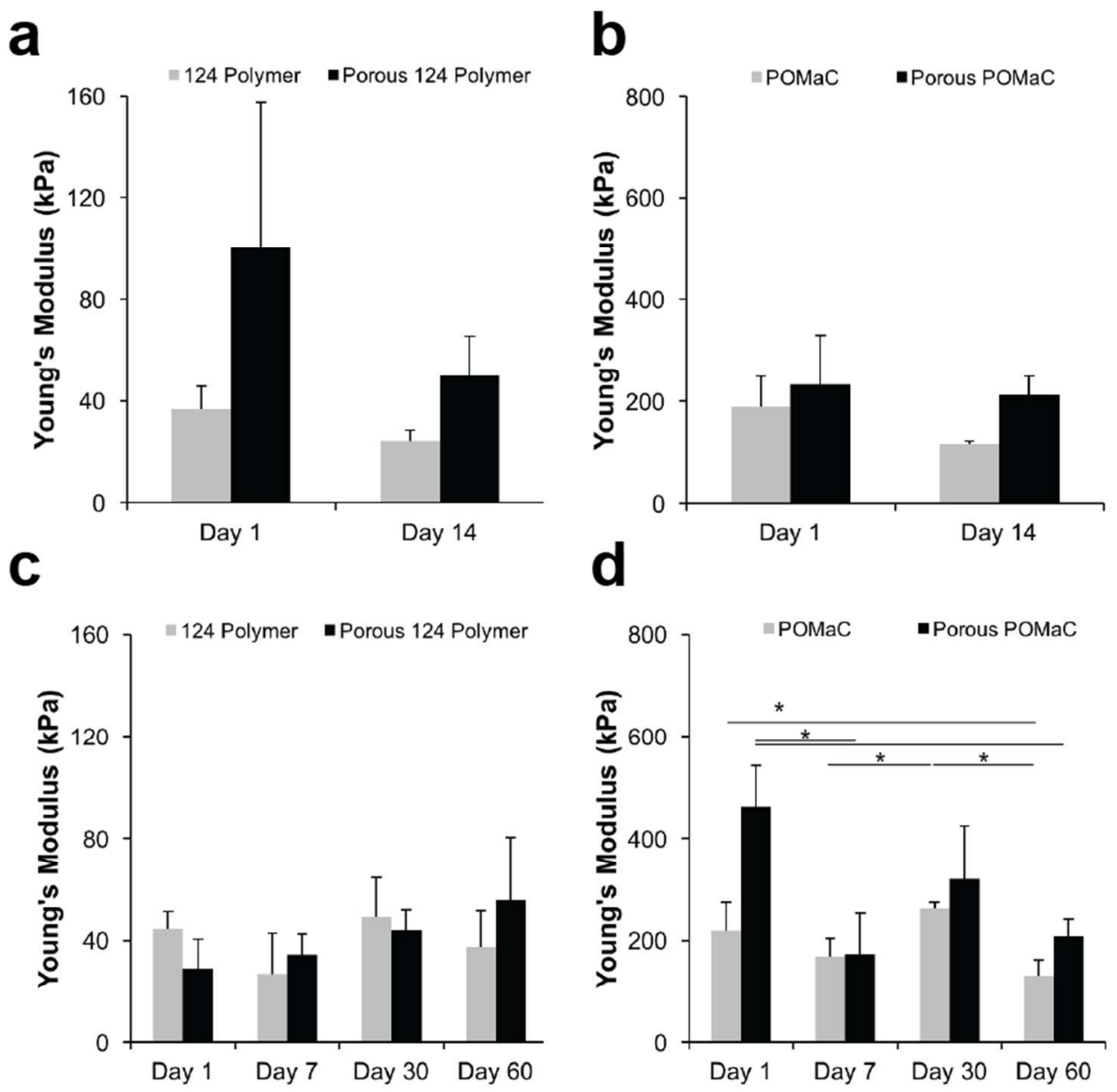

Figure S2: Change in Young's modulus in solution. In a transwell assay with neonatal rat cardiomyocytes over a 14 day period at $37^{\circ} \mathrm{C}$ change in Young's modulus was observed for photocrosslinked 124 polymer (a) and POMaC (b) both in their pure form and with $40 \%(\mathrm{~m} / \mathrm{m})$ initial porogen content $\left.{ }^{*} p<0.05, n=5\right)$. Change in Young's modulus was also assessed in phosphate buffered saline over a 60 day period at $37^{\circ} \mathrm{C}$ for photocrosslinked 124 polymer (c) and POMaC (d) in pure form and containing $40 \%(\mathrm{~m} / \mathrm{m})$ initial porogen content $\left({ }^{*} p<0.05, n=5\right)$.

\section{In vitro assessment and LDH Assay:}

Methods:

A preliminary cytotoxicity study was conducted with rat cardiac fibroblast culture on the base of 24-well tissue culture polystyrene plates (Cellstar). 124 polymer and POMaC scaffold meshes were prepared as described for other methods in this study and suspended wells with cells in neonatal rat cell culture media. Scaffold-free wells were used as control. Media was changed every two days and cells were grown for six days. Bright field images were taken to assess wells containing polymer meshes to those without. 
A similar study was conducted where crosslinked 124 polymer films were placed at the base of 24 well plates. Films were crosslinked as previously described from 124 pre-polymer, soaked in PBS first followed by $70 \%$ ethanol overnight, and washed twice in PBS. Neonatal rat cardiac fibroblasts were seeded on the films and on the base of 24-well plates as a relative control with neonatal rat cell culture media. Media was changed and collected every two days for a 10-day period. Cell-death analysis was determined from assessment of cell media using an LDH Cytotoxicity Assay Kit (Cayman Chemical Company) as per the manufacturer's instructions. The absorbance readings were compared between wells containing films and polystyrene plate controls.

Results:
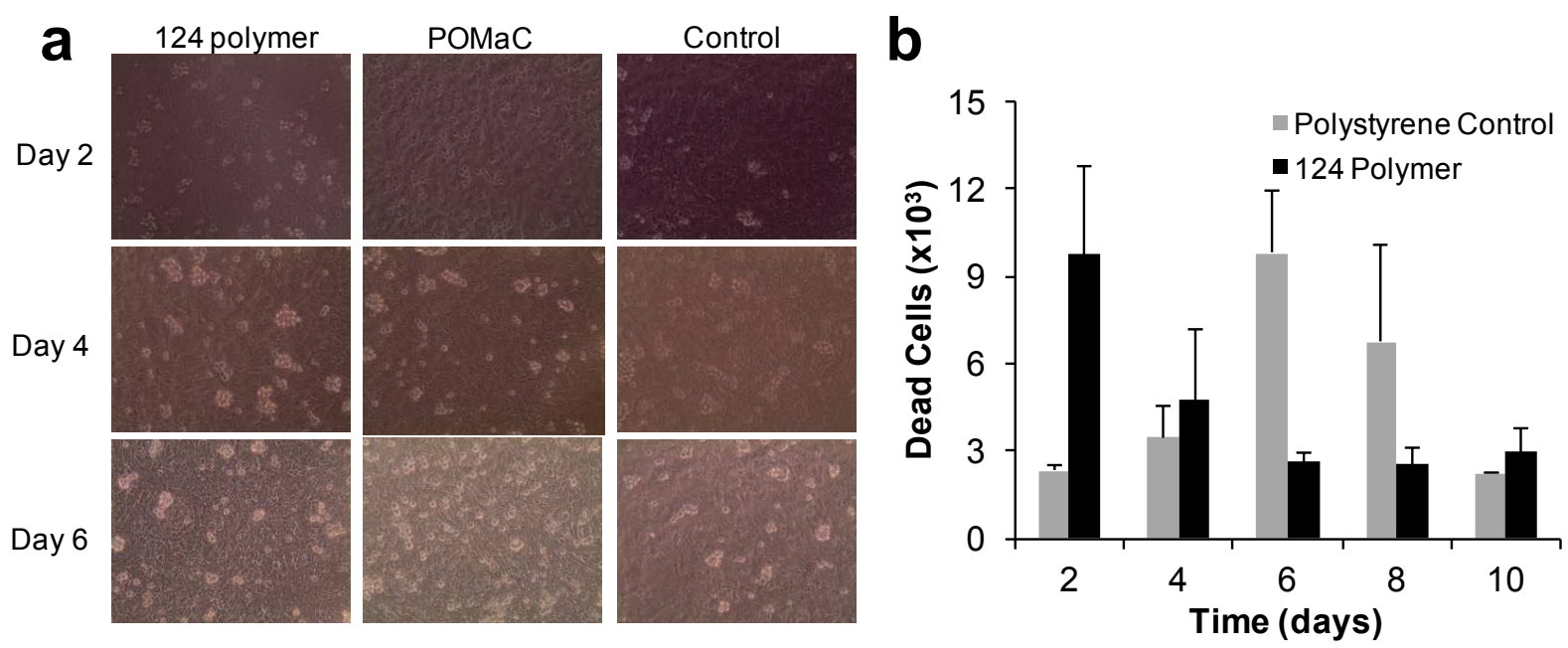

Figure S3: In vitro cytotoxicity assessment of 124 polymer. (a) Rat cardiac fibroblast cells seeded on the base of tissue culture plates with suspended polymer scaffold meshes showed no visual difference in cell viability. (b) LDH assay assessment of rat cardiac fibroblast cells seeded on 124 polymer films and in polystyrene wells as a control. 


\section{Long term in vivo assessment:}

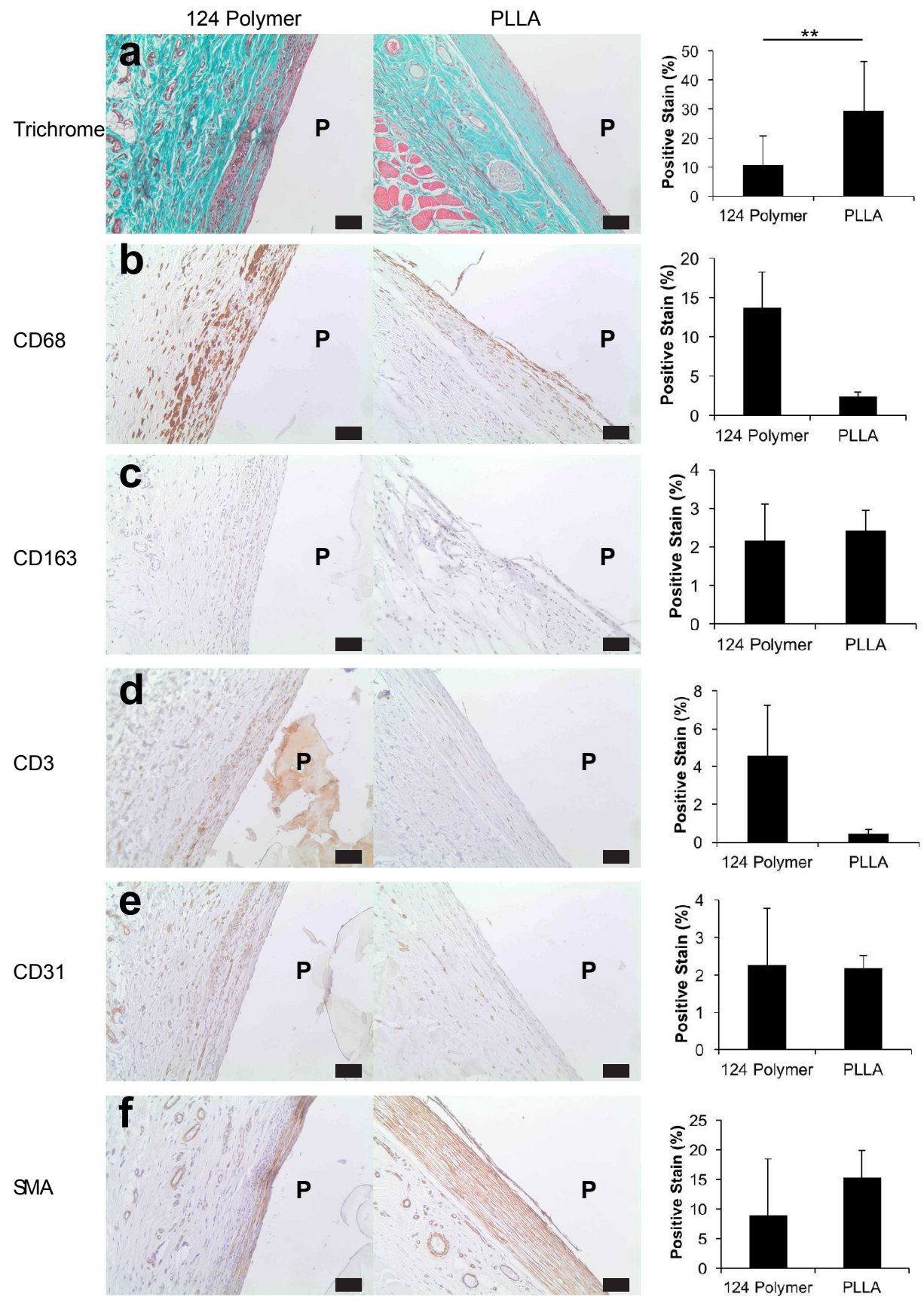

Figure S4: Photomicrographs of rat tissue explants which surrounded subcutaneous polymer discs of 124 polymer (left) and PLLA (right) 60 days post-implantation. Images were stained for specific markers. All stains were quantified as percentage of total area with a positive stain $\left({ }^{*} p<0.05,{ }^{* *} p<0.1, P=p o l y m e r\right.$ disc) (a) Masson's Trichrome staining presents less collagen deposition along the 124 polymer disc boundary in comparison to PLLA control. (b) Staining for all macrophages (CD68) and (c) M2 macrophages (CD163) shows increased total macrophage presence in the 124 polymer compared to the PLLA group. (d) T-cell recruitment (CD3) is also heightened along 124 polymer sample boundaries compared to the control but with low absolute quantities. (e-f) Staining for vascular markers that signify vascularization, CD31 and smooth muscle actin (SMA), do not present any appreciable differences. Scale bars, $25 \mu \mathrm{m}$. 


\section{Development of design of experiments model for Young's Modulus:}

We developed a model using a standard empirical modelling approach to help one determine a minimum and a maximum value in a given experimental space with as few experiments as possible. This mathematical model was developed using a standardized $2^{3}$ factorial experimental design of experiments ${ }^{1}$. This method accomplishes the goal of minimizing the number of experiments required to develop a model that relates three factors and their interactions. By performing tests at combinations of the minimum (-1) and maximum (+1) conditions, we are able to determine the main effects and interaction effects of the three parameters using 8 total experiments. Additional experiments are added at a midpoint to assess the variance of the model (Table 2).

The desired final model will have the format $\left(2^{3}\right.$ factorial design):

$$
Y=\beta_{0}+\beta_{1} A+\beta_{2} B+\beta_{3} C+\beta_{12} A B+\beta_{13} A C+\beta_{23} B C+\beta_{123} A B C
$$

We can define matrices $\mathbf{X}$ and $\mathbf{Y}$, which have the following format:

$\boldsymbol{X}=\left[\begin{array}{ccc}A_{1} & \cdots & A B C_{1} \\ \vdots & \ddots & \vdots \\ A_{n} & \cdots & A B C_{n}\end{array}\right], \mathbf{Y}=\left[\begin{array}{c}Y_{1} \\ \vdots \\ Y_{n}\end{array}\right]$

where $n$ is the number of trials.

For the development of the model for Young's Modulus seen in Figure 2, we selected the desired range of testing and coded the parameters according to Equation 1. This general equation was used to code the high and low values (Equations 2-4) for each variable, and the associated high and low values are summarized in Table 1.

We tested the Young's Modulus of 124 polymer material using the following $\mathbf{X}$ matrix conditions, comparing all combinations of the maximum and minimum values of each parameter, as well as a midpoint replicate. The $\mathrm{Y}$ matrix contains Young's Modulus $(\mathrm{kPa})$ values extracted from the stress/strain curves for each trial. 


$\mathbf{X}=\left[\begin{array}{cccccccc}1 & 1 & 1 & 1 & 1 & 1 & 1 & 1 \\ 1 & -1 & 1 & 1 & -1 & 1 & -1 & -1 \\ 1 & -1 & -1 & 1 & 1 & -1 & -1 & 1 \\ 1 & 1 & -1 & 1 & -1 & -1 & 1 & -1 \\ 1 & 1 & 1 & -1 & 1 & -1 & -1 & -1 \\ 1 & -1 & 1 & -1 & -1 & -1 & 1 & 1 \\ 1 & 1 & -1 & -1 & -1 & 1 & -1 & 1 \\ 1 & -1 & -1 & -1 & 1 & 1 & 1 & -1 \\ 1 & -\frac{1}{3} & 0 & 0 & 0 & 0 & 0 & 0 \\ 1 & -\frac{1}{3} & 0 & 0 & 0 & 0 & 0 & 0 \\ 1 & -\frac{1}{3} & 0 & 0 & 0 & 0 & 0 & 0 \\ 1 & -\frac{1}{3} & 0 & 0 & 0 & 0 & 0 & 0 \\ 1 & -\frac{1}{3} & 0 & 0 & 0 & 0 & 0 & 0 \\ 1 & -\frac{1}{3} & 0 & 0 & 0 & 0 & 0 & 0 \\ 1 & -\frac{1}{3} & 0 & 0 & 0 & 0 & 0 & 0 \\ 1 & -\frac{1}{3} & 0 & 0 & 0 & 0 & 0 & 0 \\ 1 & -\frac{1}{3} & 0 & 0 & 0 & 0 & 0 & 0 \\ 1 & -\frac{1}{3} & 0 & 0 & 0 & 0 & 0 & 0 \\ 1 & -\frac{1}{3} & 0 & 0 & 0 & 0 & 0 & 0\end{array}\right] \quad \mathbf{Y}=\left[\begin{array}{c}701.10 \\ 744.79 \\ 35.20 \\ 399.39 \\ 562.85 \\ 94.92 \\ 170.05 \\ 75.4 \\ 199.43 \\ 240.90 \\ 223.20 \\ 151.20 \\ 276.45 \\ 245.34 \\ 214.36 \\ 292.61 \\ 188.12 \\ 207.30\end{array}\right]$

The $\beta$ parameters are determined using the following equation:

$$
\begin{gathered}
\boldsymbol{\beta}=\left(X^{T} X\right)^{-\mathbf{1}} \boldsymbol{X}^{\boldsymbol{T}} \boldsymbol{Y} \\
\boldsymbol{\beta}=\left[\begin{array}{c}
\beta_{0} \\
\beta_{1} \\
\beta_{2} \\
\beta_{3} \\
\beta_{12} \\
\beta_{13} \\
\beta_{23} \\
\beta_{123}
\end{array}\right]=\left[\begin{array}{c}
302 \\
126 \\
178 \\
122 \\
-4.33 \\
-30.3 \\
74.9 \\
-97.6
\end{array}\right]
\end{gathered}
$$

Statistical testing was conducted using a student t-test on each parameter, and those with $p<0.05$ were dropped from the model (Figure $2 \mathbf{b}$ ), giving the following matrix:

$$
\boldsymbol{\beta}=\left[\begin{array}{c}
\beta_{0} \\
\beta_{1} \\
\beta_{2} \\
\beta_{3} \\
\beta_{23} \\
\beta_{123}
\end{array}\right]=\left[\begin{array}{c}
302 \\
126 \\
178 \\
122 \\
74.9 \\
-97.6
\end{array}\right]
$$

These values are used to develop the model for Young's Modulus presented in Figure 2. 


\section{Scaffold preparation technique:}

a
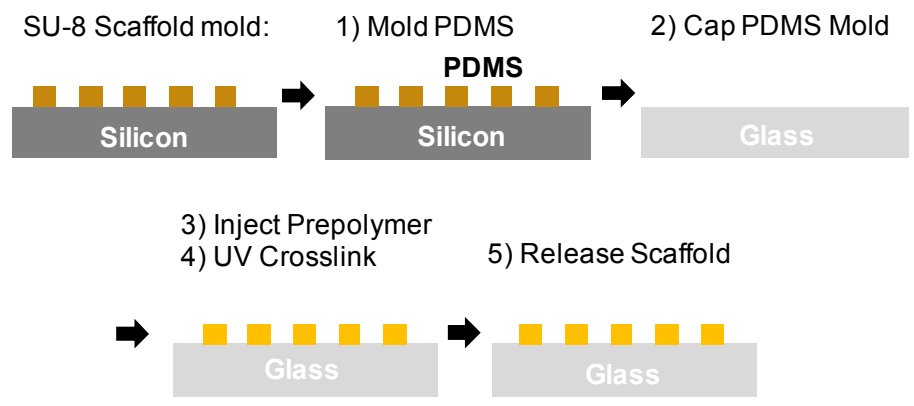

b

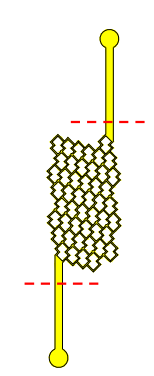

C

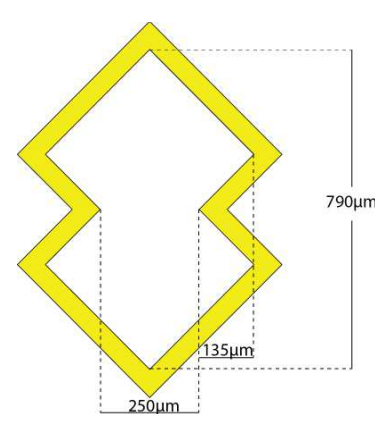

Figure S5: (a) The preparation technique for tissue mesh structures shown as a two-dimensional cross section. The SU-8 scaffold mold is prepared using photolithography techniques. A PDMS negative is poured on the mold, cured, and removed. The PDMS is capped with a glass cover slip, injected with 124 pre-polymer gel, and crosslinked under UV light. Once crosslinked, the PDMS is peeled off from the glass, leaving behind the mesh structure to then be removed under wet conditions. (b) The overall scaffold top view, which has a nominal height of $100 \mu \mathrm{m}$. The injection ports are removed (above/below the red line) before cell seeding. (c) The repeating unit dimensions, where the mesh struts have a thickness of $50 \mu \mathrm{m}$

Supplementary Movie 1: 124 polymer tissue mesh seeded with rat cardiomyocytes under electrical field stimulation

Supplementary Movie 2: 124 polymer tissue mesh seeded with rat cardiomyocytes demonstrates spontaneous beating before and after handling and maintenance of structural integrity

\section{References:}

1. Montgomery, D. C.; Runger, G. C.; Hubele, N. F., Engineering statistics. Wiley: 2011. 